\title{
HAS THE QUALITY OF REPORTING IN \\ PERIODONTOLOGY CHANGED IN 14 YEARS? A SYSTEMATIC REVIEW
}

N. Leow ${ }^{1}$, Z. Hussain ${ }^{2}$, A. Petrie ${ }^{3}$, N. Donos ${ }^{4}$ and I. Needleman ${ }^{5}$

\author{
RUNNING TITLE \\ Quality of Reporting in Periodontology
}

\section{KEY WORDS}

Clinical trials, research design, RCTs, random allocation, bias

\section{CORRESPONDING AUTHOR}

Natalie M. Leow

Unit of Periodontology,

UCL Eastman Dental Institute

256 Gray's Inn Rd, London WC1X 8LD

Tel: (+44) 0203456 1171, Fax: (+44) 02034561137

Email: n.leow@ucl.ac.uk

\section{AUTHOR AFFILIATIONS}

Unit of Periodontology ${ }^{1}$, Biostatistics ${ }^{3}$ and International Centre for EvidenceBased Oral Health ${ }^{5}$, University College London, Eastman Dental Institute, 256 Gray's Inn Road, London WC1X 8LD, United Kingdom.

Department of Restorative Dental Sciences ${ }^{2}$, Eastman Dental Hospital, 256 Gray's Inn Road, London WC1X 8LD, United Kingdom.

Clinical Oral Research Centre ${ }^{4}$, Barts and The London School of Medicine and Dentistry, Queen Mary University of London, Turner Street, London E1 2AD, United Kingdom. 


\title{
HAS THE QUALITY OF REPORTING IN PERIODONTOLOGY CHANGED
}

\section{IN 14 YEARS? A SYSTEMATIC REVIEW}

\author{
N. Leow ${ }^{1}$, Z. Hussain ${ }^{2}$, A. Petrie ${ }^{3}$, N. Donos ${ }^{4}$ and I. Needleman ${ }^{5}$
}

\section{CONFLICT OF INTEREST AND SOURCE OF FUNDING STATEMENT}

No external funding was received for this study. University College London paid salaries to NL, IN, AP and ND. NHS foundation trust paid salary to ZH. The authors declare no conflict of interest.

\section{ABSTRACT}

Quality of reporting randomised controlled trials in periodontology has been poor. Consolidated Standards of Reporting Trials guidelines and an extension for non-pharmacologic trials (CONSORT-NPE), were introduced to aid in improving this. Aims: Assess the quality of reporting in periodontology, changes over the last 14 years, and adherence to CONSORT-NPE.

Methods: RCTs in humans, published in three periodontal journals, from 2013-2015 were included. Search was conducted through Medline, Embase and hand searching. Results: 173 full text articles included. Two reviewers screened for reporting quality (kappa $=0.69,95 \% \mathrm{Cl} 0.60-0.76$ ). $84 \%$ of studies $(n=145)$ described randomisation methods, $74 \%(n=128)$ highlighted examiner blinding, and $87 \%(n=151)$ accounted for patients at study conclusion. Patient and caregiver blinding was addressed in $50 \%(n=70)$ and $50 \%(n=27)$ of studies, respectively. 64\% ( $n=110)$ described adequate allocation concealment. Compared with Montenegro et al. 2002, improvements seen in describing randomisation (2002,16.5\%; 2016,84\%), allocation concealment (2002,6.5\%; 2016,64\%), caregiver masking (2002,17\%; 2015,50\%).

CONSORT-NPE; $62 \%(n=107)$ had detailed explanations of all treatments, $88 \%(n=152)$ lacked protocols for adherence of caregivers' to an intervention. Only $17 \%(n=29)$ described caregivers' expertise and case volume.

Conclusions: Substantial improvements have occurred. Attention is required 
for statistical analysis of patient losses and masking. CONSORT-NPE aspects were poorly reported.

\section{CLINICAL RELEVANCE}

Scientific rationale for the study: Reporting quality of RCTs is pivotal to determine potential sources of bias. If poor reporting quality reflects actual study conduct, the size of treatment effects, ability to repeat a study, and ability to make accurate clinical decisions based on sound evidence is put into question.

Principal findings: Substantial improvements in reporting have occurred, however for RCTs, the relevant extension (CONSORT-NPE) is poorly reported on.

Practical implications: The present findings may encourage authors of RCTs to report more comprehensively with the use of CONSORT and CONSORTNPE. Additionally, journal editors may guide authors to appropriate CONSORT extensions prior to submission of an article. 


\section{INTRODUCTION}

Randomised controlled trials (RCTs) are considered the gold standard for research on interventions in healthcare (Sackett et al., 2000). Good quality reporting of RCTs is important to assess the effect of an intervention and to reliably appraise the relevance and validity of study findings. In some disciplines of dentistry, this quality has been reported as being problematic (Al - Namankany et al., 2009, Montenegro et al., 2002, Kunz et al., 2007). Furthermore, subsequent to the publication of the initial Consolidated Standards of Reporting Trials statement (CONSORT) (Begg et al., 1996), reviews have shown the quality of reporting of RCTs remains below an acceptable level (AI - Namankany et al., 2009, Tu et al., 2006, Froud et al., 2012, Hopewell et al., 2008).

Quality assessment of RCTs is important to, 'estimate that results are an accurate estimate of the truth', (Moher et al., 2001) and to clarify the size of treatment effect of an intervention. Clear and thorough reporting of a study is therefore extremely important, as, inadequate descriptions make repeating the trial very difficult, if not impossible. Additionally, clinicians may not know how to appropriately deliver a beneficial intervention (Cook et al., 2013). To assist clear and transparent reporting of RCTs, the original CONSORT statement was published (Begg et al., 1996) and has been revised twice since (Moher et al., 2001, Schulz et al., 2010). CONSORT was originally devised for use with pharmacological studies with a, 'standard' two group parallel design. To account for studies that do not fall into this category, the, 'non- 
pharmacologic' extension (CONSORT-NPE) was published (Boutron et al., 2008). This was to aid and encourage full reporting of details of operative procedures. The extension has particular relevance to periodontal RCTs, many of which investigate operative or surgical interventions.

Montenegro et al. (2002) conducted a systematic review of RCTs published in three periodontal journals from 1996 to 1998 . They found that most studies did not meet recommendations on reported study quality. In fact, only $17 \%$ reported adequate randomisation and $7 \%$ adequate concealment methods. If these findings represented actual study conduct, they represented a significant risk of bias.

More than a decade has passed since this comprehensive assessment of reported quality of RCTs in the periodontal literature (Montenegro et al., 2002). During this time, the CONSORT statement has been adopted by numerous journals (Needleman et al., 2008) and the CONSORT-NPE has been published (Boutron et al., 2008). Since CONSORT has been available for some years, we were interested to investigate whether quality of reporting has improved since our last assessment. The aims of the present systematic review were, a) to assess the current quality of reporting RCTs in periodontology, b) to report whether the reported quality has improved over the last 14 years, and, c) to assess adherence of periodontal RCTs to the CONSORT-NPE within the three main specialist journals in periodontology.

\section{MATERIALS AND METHODS}

Protocol Development 
This systematic review followed much of the methodology of Montenegro et al. (2002) in order to allow comparison of results. The inclusion criteria were RCTs of interventions published in Journal of Clinical Periodontology, Journal of Periodontology, and Journal of Periodontal Research. Included trials were conducted in humans and published between $1^{\text {st }}$ January 2013 and $31^{\text {st }}$ December 2015 (inclusive). The initial review protocol was devised, including aim(s), search strategy, inclusion criteria, data abstraction and data analysis.

\section{Search Strategy}

Electronic searches of both MEDLINE $\left(\mathrm{Ovid}^{\circledR}\right)$ and EMBASE $\left(\mathrm{Ovid}^{\circledR}\right)$ were conducted for RCTs published between 2013 and 2015 (inclusive) by a single author (NL). The search was limited to the Journal of Clinical Periodontology, Journal of Periodontology, and Journal of Periodontal Research. Additionally, hand searching of each journal was carried out. Search terms for RCTs were implemented according to recommendations outlined by the, 'Cochrane Handbook for Systematic Reviews of Interventions' (Higgins and Green, 2011).

\section{Quality Assessment}

Twenty-five key components derived from the CONSORT statement and CONSORT-NPE was assessed (Boutron et al., 2008, Schulz et al., 2010). This systematic review looked at those elements reported by Montenegro et al. (2002), along with additional areas pertinent to the CONSORT-NPE. Definitions for randomisation, adequate allocation concealment and blinding were used as per the previous study also. The CONSORT-NPE guidance includes a number of extensions to the CONSORT 
checklist in order to gain more specific information, the addition of one item, and modification of the flow diagram (Boutron et al., 2008). Rather than a standalone guidance, the extension is recommended for use as an addendum to the main CONSORT statement (Schulz et al., 2010). Table S1 displays a summary of key CONSORT-NPE aspects.

Descriptions of the CONSORT-NPE were derived from (Boutron et al., 2008) . The following descriptions were used;

- Precise details of both the experimental treatment and comparator. A detailed description of the experimental treatment should be given so as to enable fair study comparison and reproducibility. Additionally, the comparator should be described in detail, even if it is deemed as usual care. This is so that the intensity of usual care and the experimental intervention can be compared.

- Description of the different components of the intervention and, when applicable, description of the procedure for tailoring the intervention to individual participants. Particularly when the interventions were complex, each component should be described in detail as this may influence the estimated effect of treatment. Reporting of how the intervention was tailored to account for co-morbid conditions or participant tolerance should be described.

- Details of standardisation of interventions. This becomes particularly relevant for multicentre trials so that interventions are uniform and comparable across the trial. Standardisation might be as simple as informing providers to carry out treatment as they normally would or require particular training and/or certification of certain techniques or procedures. 
- Descriptions of care providers and centres in each group. Applicable for multi-centre trials, information should be given on the number of centres involved in the trial and number of care providers in each group. Also, the distribution of participants treated by each care provider.

-Take into account the choice of the comparator, lack of or partial blinding, and unequal expertise of care providers or centres in each group. This would include any differences that would impact on generalisability of the results. Examples of this would be a particular health care system, selection of health care centres and care providers. Furthermore, it is recommended authors indicate whether an intervention is likely to perform the same in all settings.

\section{Screening and Data Abstraction}

Two reviewers carried out screening of titles and abstracts from the search in duplicate and independently. Full text articles were subsequently obtained for all potentially eligible studies for screening by the same reviewers and according to the same criteria, and, upon agreement of final included studies, data abstraction was completed. All stages of screening and data abstraction were carried out independently by the same two reviewers (NL \& ZH).

Kappa scores calculated agreement, and disagreement was resolved by discussion. A single reviewer (NL) performed data entry for descriptive statistics (IBM SPSS Version 21.0, IBM Corp, Armonk, NY, USA).

\section{RESULTS}

\section{Search}


The initial search found 492 articles for screening. From these, 173 full text articles were included for data abstraction (Table S2). The distribution according to journals was: Journal of Clinical Periodontology, 81 (47\%), Journal of Periodontology, 75 (43\%) and Journal of Periodontal Research, 17 (10\%). Figure 1. displays the flow of articles through the screening process. Studies were excluded after full-text for the following reasons: not an RCT (13), additional publication of an already included study population (8) and duplicate (1). Table S3 shows studies that were excluded and reasons.

Figure 1 to be inserted here

\section{Study Quality Components}

\section{Inter-reviewer agreement}

Agreement for screening of titles and abstracts was 0.87 (95\% Cl 0.82-0.91), and for full text articles was 0.82 (95\% Cl 0.67-0.94). Mean inter-reviewer agreement for data abstraction of the random selection of articles was good at 0.69 (95\% Cl 0.60-0.76) (Landis and Koch, 1977). Table 1 displays kappa values for a number of quality components.

\section{Current reported quality (Table 2)}

The results show that all 173 studies (100\%) were described as being randomised with 145 trials (84\%) clearly describing randomisation methods. We judged allocation concealment as adequate in 110 trials (64\%). Almost three quarters of included studies (128 studies, 74\%) outlined adequate examiner blinding methods (limited to studies where examiner blinding might be possible). Blinding of patients and caregivers was poorly addressed with 
only $50 \%$ of studies (for both aspects) providing a description (where deemed possible). Finally, 151 studies (87\%) accounted for all patients at the end of each trial whilst only 35 trials (32\%) appeared to account for patient losses in statistical analyses.

\section{Comparison to Montenegro et al. 2002}

Overall, there has been a substantial improvement in the reported quality of RCTs in periodontology compared to 2002 (Table 2). This is particularly evident for adequate randomisation methods (2002, 16.5\%; 2016, 84\%), adequate allocation concealment (2002, 6.5\%; 2016, 64\%) and accounting for all patients at the end of the study (2002, 56\%; 2016, 87\%). Examiner masking (blinding) also showed improvement (2002, 55\%; 2016, 74\%), as did patient blinding (where deemed possible) (2002, 24\%; 2016, 50\%).

\section{CONSORT- NPE (Table 3)}

\section{Description of interventions}

We found that 107 studies (62\%) described precise details of both the experimental treatment and comparator. Furthermore, 108 RCTs (62\%) gave a description of the different components of the interventions, and if applicable, tailoring to individual patients. Almost half the studies did not report on standardisation of care (80 studies, 46\%) and 152 trials (88\%) did not describe any detail on how adherence of care providers with the protocol was either assessed or enhanced (e.g. review of case report forms or videotapes). 


\section{Description of care providers}

Only 29 studies (17\%) provided some description of care providers with regard to case volume, qualification, expertise or the number of patients treated by each provider.

\section{Aspects of Generalisability of Study}

The applicability and usefulness of a clinical trial's results is undoubtedly an important aspect to consider. For non-pharmacologic trials, the 'external validity' may be assessed according to a number of factors that includes: describing (in detail) all interventions provided, both experimental and control (comparator); outlining methods of blinding (e.g. if full blinding not possible, then blinding of participants to the study hypothesis, 'partial blinding', could be considered) and; a description of each caregiver's experience and how the care provided was assessed (expertise) (Boutron et al., 2008, Cook et al., 2013). Almost one third of studies (48 trials, $28 \%$ ) did not address aspects of describing the comparator, partial blinding and expertise of caregivers within their trials. This undoubtedly has implications for generalisability of those studies.

\section{DISCUSSION}

\section{Key Findings}

This systematic review has shown that in the past 14 years, the reported quality of RCTs in periodontology has substantially improved. All CONSORT quality aspects have improved since 2002. Methods of randomisation, allocation concealment and care provider masking have improved most. However, considering the adherence to aspects of the more recently 
introduced non-pharmacologic extension of CONSORT, which is particularly relevant to periodontology, RCTs frequently do not meet the recommended quality of reporting. Particular areas requiring attention; specific descriptors of care provider (s), and adherence of care provider(s) to a protocol.

\section{Comparison to Other Studies}

The improvements in reporting of RCTs in periodontology following adoption of the CONSORT statement are similar to studies in the biomedical literature (Gray et al., 2012, Turner et al., 2012). A recent update (Turner et al., 2012) to a systematic review in medical journals from 2005 to 2010 (Plint et al., 2006) found that reporting of randomisation sequence generation, sample size and allocation concealment had improved, but less change was seen with reporting of participant flow, and blinding of participants and data analysts. This is similar to our findings. Adoption of CONSORT guidelines appeared to have minimal effect on reporting of all categories of masking, and participant flow (Plint et al., 2006, Turner et al., 2012), which is similar to RCTs in periodontology.

In relation to CONSORT-NPE, Gray et al. (2012) assessed adherence of trials of an operative intervention to both the CONSORT statement and the non-pharmacologic extension, before and after the extension was published. This group found a significant improvement in the mean score from 20042010 (95\% Cl: 3.61-4.29, $\mathrm{p}<0.001)$, however noted that these results were more related to the original CONSORT items rather than the CONSORT-NPE items. In fact, the authors found that the latter was still poorly reported in 
2010. Our study agrees with these findings for RCTs in periodontology. Journals included in this review did not, 'require' a CONSORT-NPE checklist for submission of a paper, which provides a possible explanation for the lack of adherence found.

Adequate randomisation and allocation concealment are key factors that determine risk of bias of a trial and therefore confidence in an estimate of effect. A review of $127 \mathrm{RCTs}$ (contained in 11 meta-analyses), reported that inadequate allocation concealment was associated with an increased or exaggerated estimate of benefit of $37 \%(R O R=0.63,0.45-0.88)$ (Moher et al., 1998) compared with studies at low risk of bias. This exaggerated estimate of benefit has been reported previously (Schulz et al., 1995) and has since been confirmed (Turner et al., 2012). Therefore, although substantially improved since 2002, the inadequate or unclear reporting of allocation concealment in $36 \%$ of trials in our review is a serious concern as we are left with substantial uncertainty about the study outcomes, particularly if this reflects actual study conduct.

\section{Strengths and Limitations}

This study sought to look at several aspects of quality assurance, in line with CONSORT recommendations. Despite endorsement of these guidelines by many high impact medical and dental journals (Hopewell et al., 2008, Needleman et al., 2008), limited knowledge exists on the extent of adherence of individual papers to these guidelines, particularly in dentistry. Furthermore, to our knowledge, this is the first study to look at the non-pharmacologic extension in regard to RCTs in periodontology or oral health. 
A limitation of this study is that we looked only at limited quality criteria. Other aspects may also be important such as case definition, statistical analysis and study design for superiority or equivalence trials (Tu et al., 2006).

Furthermore, our review is limited to three specialist periodontal journals written in English, therefore is not representative of all studies published in periodontology, nor those written in other languages.

Studies were included if they reported on a population whereby specifics of methodology or population were published previously. Often authors would refer to a previous publication for details. This would influence our results as the previous publications were often published prior to endorsement of CONSORT guidelines by journals.

\section{Implications}

Although the journals included in this research endorse the CONSORT checklist, none currently direct authors to the range of different versions that may be appropriate to particular study designs including cluster, noninferiority, pragmatic and patient reported outcome trials. We would encourage oral health journals to both include the current range of CONSORT statements and raise awareness of what these offer to the academic community. All can be found at http://www.equator-network.org. Since so many periodontology trials are operative, endorsing and requiring the CONSORT-NPE extension for submission of a publication seems particularly relevant to quality improvement with a further benefit of enhancing the potential to synthesise trials more effectively in systematic reviews. 


\section{CONCLUSION}

Within the limitations of this study, we have found substantial improvement in the quality of reporting of RCTs in periodontology since our the last review (Montenegro et al., 2002). Further improvement in reporting of key aspects of methodology which are associated with the greatest risk of bias of trials is a priority. The period of observation of this study coincides with endorsement of CONSORT guidelines by the journals in this report. Items specific to the newer non-pharmacologic extension, which is particularly relevant to operative studies however, are under-reported. Employment of the nonpharmacologic CONSORT extension would help to improve the quality of reporting a substantial proportion of RCTs in periodontology.

\section{Supporting Information}

Table S1. Summary table of CONSORT-NPE

Table S2. Excluded studies

Table S3. Included studies 


\section{REFERENCES}

AL - NAMANKANY, A., ASHLEY, P., MOLES, D. \& PAREKH, S. 2009.

Assessment of the quality of reporting of randomized clinical trials in paediatric dentistry journals. International Journal of Paediatric Dentistry, 19, 318-324.

BEGG, C., CHO, M., EASTWOOD, S., HORTON, R., MOHER, D., OLKIN, I., PITKIN, R., RENNIE, D., SCHULZ, K. F. \& SIMEL, D. 1996. Improving the quality of reporting of randomized controlled trials: the CONSORT statement. Journal of the American Medical Association, 276, 637-639. BOUTRON, I., MOHER, D., ALTMAN, D. G., SCHULZ, K. F. \& RAVAUD, P. 2008. Extending the CONSORT statement to randomized trials of nonpharmacologic treatment: explanation and elaboration. Annals of Internal Medicine, 148, 295-309.

COOK, A., DOUET, L. \& BOUTRON, I. 2013. Descriptions of nonpharmacological interventions in clinical trials. British Medical Journal, 347, f5212.

FROUD, R., ELDRIDGE, S., DIAZ ORDAZ, K., MARINHO, V. C. C. \& DONNER, A. 2012. Quality of cluster randomized controlled trials in oral health: a systematic review of reports published between 2005 and 2009. Community Dentistry and Oral Epidemiology, 40, 3-14.

GRAY, R., SULLIVAN, M., ALTMAN, D. \& GORDON-WEEKS, A. 2012. Adherence of trials of operative intervention to the CONSORT statement extension for non-pharmacological treatments: a 
comparative before and after study. Annals of the Royal College of Surgeons of England, 94, 388-394.

HIGGINS, J. \& GREEN, S. (eds.) 2011. Cochrane handbook for systematic reviews of interventions: The Cochrane Collaboration.

HOPEWELL, S., ALTMAN, D. G., MOHER, D. \& SCHULZ, K. F. 2008. Endorsement of the CONSORT Statement by high impact factor medical journals: a survey of journal editors and journal 'Instructions to Authors'. Trials, 9, 20.

KUNZ, R., VIST, G. \& OXMAN, A. D. 2007. Randomisation to protect against selection bias in healthcare trials. Cochrane Database of Systematic Reviews, 2.

LANDIS, J. R. \& KOCH, G. G. 1977. The measurement of observer agreement for categorical data. Biometrics, 159-174.

MOHER, D., JONES, A., COOK, D. J., JADAD, A. R., MOHER, M., TUGWELL, P. \& KLASSEN, T. P. 1998. Does quality of reports of randomised trials affect estimates of intervention efficacy reported in meta-analyses? The Lancet, 352, 609-613.

MOHER, D., SCHULZ, K. F., ALTMAN, D. G. \& GROUP, C. 2001. The CONSORT statement: revised recommendations for improving the quality of reports of parallel-group randomised trials. The Lancet, 357, 1191-1194.

MONTENEGRO, R., NEEDLEMAN, I., MOLES, D. \& TONETTI, M. 2002. Quality of RCTs in periodontology—a systematic review. Journal of Dental Research, 81, 866-870.

NEEDLEMAN, I., MOHER, D., ALTMAN, D., SCHULZ, K., MOLES, D. \& WORTHINGTON, H. 2008. Improving the clarity and transparency of 
reporting health research: a shared obligation and responsibility. Journal of Dental Research, 87, 894-895.

PLINT, A. C., MOHER, D., MORRISON, A. \& SCHULZ, K. 2006. Does the CONSORT checklist improve the quality of reports of randomised controlled trials? A systematic review. Medical Journal of Australia, $185,263-267$.

SACKETT, D., STRAUS, S., RICHARDSON, S., ROSENBERG, W. \& HAYNES, B. 2000. Evidence-based medicine: how to practice and teach EBM, London, Churchill Livingstone.

SCHULZ, K. F., ALTMAN, D. G. \& MOHER, D. 2010. CONSORT 2010 statement: updated guidelines for reporting parallel group randomised trials. BioMed Central Medicine, 8, 1.

SCHULZ, K. F., CHALMERS, I., HAYES, R. J. \& ALTMAN, D. G. 1995. Empirical evidence of bias: dimensions of methodological quality associated with estimates of treatment effects in controlled trials. Journal of the American Medical Association, 273, 408-412.

TU, Y. K., MADDICK, I., KELLETT, M., CLEREHUGH, V. \& GILTHORPE, M. S. 2006. Evaluating the quality of active - control trials in periodontal research. Journal of Clinical Periodontology, 33, 151-156.

TURNER, L., SHAMSEER, L., ALTMAN, D. G., WEEKS, L., PETERS, J., KOBER, T., DIAS, S., SCHULZ, K. F., PLINT, A. C. \& MOHER, D. 2012. Consolidated standards of reporting trials (CONSORT) and the completeness of reporting of randomised controlled trials (RCTs) published in medical journals. Cochrane Database of Systematic Reviews, 11. 
Has The Quality Of Reporting In Periodontology Changed In 14 Years? A Systematic Review 


\section{TABLES}

Table 1. Inter-Examiner Agreement for Quality Assessment (Kappa Score).

Kappa Score

\section{CONSORT}

Described as randomised

0.80

Randomisation method

0.68

Allocation concealment method adequate

0.78

Patient blinding

0.75

Caregiver blinding

0.59

Examiner blinding

0.76

All patients accounted for at end of study

0.78

Analysis accounts for patient losses

0.67

\section{CONSORT- NPE}

How the interventions were standardised

0.76

Description of care providers

Account for choice of comparator, lack of partial blinding, and

0.72 unequal expertise of care providers/ centers 
Table 2. Comparison of Quality Assessment of RCTs.

\begin{tabular}{|c|c|c|c|c|c|}
\hline & & $\begin{array}{c}\text { Yes/ } \\
\text { Adequate } \\
\mathrm{n}(\%)\end{array}$ & $\begin{array}{c}\mathrm{No} / \\
\text { Inadequate } \\
\mathrm{n}(\%)\end{array}$ & $\begin{array}{l}\text { Unclear } \\
\text { n (\%) }\end{array}$ & $\begin{array}{c}\text { Not } \\
\text { Applicable } \\
\text { n (\%) }\end{array}$ \\
\hline Described as & Montenegro et al., (2002) & $161(91)$ & $15(8.5)$ & $1(0.5)$ & - \\
\hline randomised & Current study & $173(100)$ & - & - & - \\
\hline Randomisation & Montenegro et al., (2002) & $29(16.5)$ & $1(0.5)$ & $147(83)$ & - \\
\hline methods adequate & Current study & $145(84)$ & $3(2)$ & $25(14)$ & - \\
\hline Allocation & Montenegro et al., (2002) & $12(6.5)$ & $1(0.5)$ & $164(93)$ & - \\
\hline $\begin{array}{c}\text { concealment } \\
\text { method adequate }\end{array}$ & Current study & $110(64)$ & $2(1)$ & $61(35)$ & - \\
\hline \multirow[t]{2}{*}{ Patient blinding } & Montenegro et al., (2002) & $42(24)$ & $77(43)$ & $58(33)$ & - \\
\hline & Current study & $70\left(50^{\star}\right)$ & $36\left(26^{\star}\right)$ & $33\left(24^{\star}\right)$ & $34(20)$ \\
\hline \multirow[t]{2}{*}{ Caregiver blinding } & Montenegro et al., (2002) & $26\left(17^{\star}\right)$ & $84\left(57^{\star}\right)$ & $38\left(26^{\star}\right)$ & $29(16)$ \\
\hline & Current study & $27\left(50^{\star}\right)$ & $18\left(33^{\star}\right)$ & $8\left(15^{\star}\right)$ & $120(69)$ \\
\hline \multirow[t]{2}{*}{ Examiner blinding } & Montenegro et al., (2002) & $97(55)$ & $12(7)$ & $68(38)$ & - \\
\hline & Current study & $128(74)$ & $14(8)$ & $31(18)$ & - \\
\hline All patients & Montenegro et al., (2002) & $100(56)$ & $25(14)$ & $52(30)$ & - \\
\hline $\begin{array}{l}\text { accounted for at } \\
\text { end of study }\end{array}$ & Current study & $151(87)$ & $7(4)$ & $15(9)$ & - \\
\hline Analysis accounts & Montenegro et al., (2002) & $11\left(11^{\star}\right)$ & $33\left(33^{\star}\right)$ & $57\left(56^{\star}\right)$ & $76(43)$ \\
\hline for patient losses & Current study & $35\left(32^{\star}\right)$ & $69\left(63^{\star}\right)$ & $5\left(5^{\star}\right)$ & $64(37)$ \\
\hline
\end{tabular}


Has The Quality Of Reporting In Periodontology Changed In 14 Years? A Systematic Review

Table 3. Items Assessed According to the CONSORT-NPE.

\begin{tabular}{|c|c|c|c|c|}
\hline & $\begin{array}{c}\text { Yes/ } \\
\text { Adequate } \\
\mathrm{n}(\%)\end{array}$ & $\begin{array}{c}\mathrm{No} / \\
\text { Inadequate } \\
\mathrm{n}(\%)\end{array}$ & $\begin{array}{l}\text { Unclear } \\
\text { n (\%) }\end{array}$ & $\begin{array}{c}\text { Not } \\
\text { Applicable } \\
\mathrm{n}(\%)\end{array}$ \\
\hline $\begin{array}{l}\text { Precise details of both experimental } \\
\text { treatment and comparator }\end{array}$ & $107(62)$ & $66(38)$ & - & - \\
\hline $\begin{array}{l}\text { Different components of intervention } \\
\text { and tailoring to participants }\end{array}$ & $108(62)$ & $65(38)$ & - & - \\
\hline How interventions were standardised & $92(53)$ & $80(46)$ & $1(1)$ & - \\
\hline $\begin{array}{c}\text { Adherence of care providers to } \\
\text { protocol described }\end{array}$ & $21(12)$ & $152(88)$ & - & - \\
\hline $\begin{array}{l}\text { Description of care providers (e.g. } \\
\text { case volume, qualification, expertise) }\end{array}$ & $29(17)$ & $131(76)$ & $13(8)$ & - \\
\hline $\begin{array}{l}\text { Description of generalisability } \\
\text { according to comparators, care } \\
\text { providers, centers. }\end{array}$ & $125(72)$ & $48(28)$ & - & - \\
\hline
\end{tabular}

